\title{
Burial depth of anode affected the bacterial community structure of sediment microbial fuel cells
}

\author{
Yi-cheng $\mathrm{Wu}^{1,3}$, Hong-jie Wu${ }^{1}$, Hai-yan $\mathrm{Fu}^{1}$, Zhineng Dai ${ }^{1}$, Ze-jie Wang $^{{ }^{\dagger}}$ \\ ${ }^{1}$ Fujian Engineering and Research Center of Rural Sewage Treatment and Water Safety, Xiamen University of Technology, Xiamen, 361024, China \\ ${ }^{2}$ College of Environmental Science and Engineering, Qilu University of Technology (Shandong Academy of Sciences), Jinan, 250353, China \\ ${ }^{3}$ Key Laboratory of Urban Pollutant Conversion, Institute of Urban Environment, Chinese Academy of Sciences, Xiamen, 361021, China
}

\begin{abstract}
Sediment microbial fuel cells (SMFCs) are attractive devices to in situ power environmental monitoring sensors and bioremediate contaminated soils/sediments. Burial depth of the anode was verified to affect the performance of SMFCs. The present research evaluated the differences in microbial community structure of anodic biofilms located at different depth. It was demonstrated that both microbial diversity and community structure of anodic biofilms were influenced by the depth of anode location. Microbial diversity decreased with increased anodic depth. The number of the operational taxonomic units (OTUs) was determined as 1438 at the anode depth of $5 \mathrm{~cm}$, which reduced to 1275 and 1005 at $10 \mathrm{~cm}$ and $15 \mathrm{~cm}$, respectively. Cluster analysis revealed that microbial communities of $5 \mathrm{~cm}$ and $10 \mathrm{~cm}$ were clustered together, separated from the original sediment and $15 \mathrm{~cm}$. Proteobacteria was the predominant phylum in all samples, followed by Bacteroidetes and Firmicutes. Betaand Gamma-proteobacteria were the most abundant classes. A total of 23 OTUs showed high identity to 16S rRNA gene of exoelectrogens such as Geobacter and Pseudomonas. The present results provided insights into the effects of anode depth on the performance of SMFC from the perspectives of microbial community structure.
\end{abstract}

Keywords: Biofilm, Burial depth, Microbial community structure, Sediment microbial fuel cell, 454 pyrosequencing

\section{Introduction}

Sediment microbial fuel cells (SMFCs) are attractive bioelectrochemical technologies that produce electricity from organic matter in sediment via metabolism of exoelectrogens [1, 2]. SMFCs attract increasing attentions as power sources to drive remote sensors to monitor environmental qualities of rivers, lakes and marine with the advantages of low cost maintenance and long-term stability [3-7]. Moreover, SMFCs have caught much attention as an effective technology to in situ remediate contaminated soil/sediment by organic or inorganic matters, via enhanced extracellular electron transfer process through accumulation of exoelectrogens [8-10].

For a typical configuration of SMFCs, the cathode is generally floating in the water layer to accept electron donors such as dissolved oxygen, while the anode is embedded in the sediment to accumulate anaerobic exoelectrogens on the anode surface [11-13]. Previous studies revealed that sediment characteristics, i.e. the concentration of $\mathrm{O}_{2}, \mathrm{NO}_{3}^{-}, \mathrm{SO}_{4}{ }^{2-}$, varied with the depth of the sediment [14, 15], accompanied with different types and physiological characteristics of microorganisms. Therefore, the depth of anode location in SMFCs was verified as an important factor affecting the electric power generation of SMFCs [1, 16]. Exoelectrogens are a key component of SMFCs [17]. Their relative abundance in anodic biofilm and electro-activity affect greatly the performance of SMFCs in terms of electric energy output and contaminants removal. Couples of research analyzed the microbial community structure of anodic biofilm in SMFCs [18, 19], while few research paid attentions to the influences of burial depth of anode on microbial diversity.

Knowledge on microbial communities of anodic biofilms with different burial depth is limited by the use of clone library methods with low sequencing depth [20]. The sequencing analysis was conducted with 454 pyrosequencing to deeply understand the microbial community structure [21-23]. In the present study, the performance of SMFCs with different anode burial depth was recorded and analyzed. The microbial diversity and exoelectrogens of anodic biofilms were

Received August 26, 2019 Accepted November 22, 2019

${ }^{\dagger}$ Corresponding author

Email: wangzejie@qlu.edu.cn

Tel: +86-531-89631680 Fax: +86-531-89631680 
revealed with the aid of 454 pyrosequencing to obtain insight into the regulation of anode burial depth on the performance of SMFCs.

\section{Material and Methods}

\subsection{Sediment and Water Samples}

Both sediment and water were sampled from a pond in Xiamen city, Fujian province. Sediment was gently separated by hand and passed through a $0.5 \mathrm{~cm}$ mesh to remove coarse debris, and then thoroughly mixed using a mechanical stirrer to ensure that the sediment was homogeneous.

\subsection{SMFCs Construction and Operation}

The SMFC reactor was a rectangular container $(40 \mathrm{~cm} \times 25 \mathrm{~cm} \times 60$ $\mathrm{cm}$ ) (Fig. 1). The sediment layer was $20 \mathrm{~cm}$ thick, covered by water layer of $7 \mathrm{~cm}$ deep. Both anode and cathode were made of graphite felt with a thickness of $0.5 \mathrm{~cm}$. Three anodes with the same size of $4 \mathrm{~cm} \times 6 \mathrm{~cm}$ were buried at 5,10 and $15 \mathrm{~cm}$ deep in the sediment, labeled as SMFC-5, SMFC-10 and SMFC-15, respectively. The cathode $(20 \mathrm{~cm} \times 12 \mathrm{~cm})$ was placed floating in the water layer and shared by the anodes. The shared cathode was connected to the anodes across an external resistance of $1000 \Omega$ via titanium wire to form SMFC-5, SMFC-10 and SMFC-15. Voltage across the external resistances was recorded at $5 \mathrm{~min}$ intervals using a digital data acquisition system (Keithley Instruments 2700, USA).

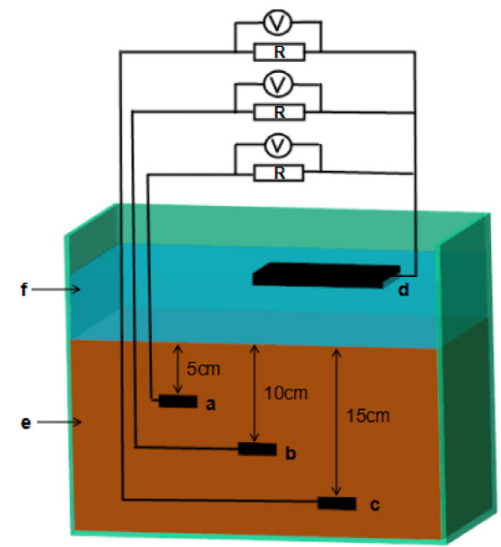

Fig. 1. The schematic of the SMFCs construction. (a), (b), and (c) indicate the anodes of the SMFC-5, SMFC-10 and SMFC-15, respectively. (d) The shared cathode of the SMFCs, (e) Sediment, (f) Water layer.

\subsection{Microbial Analysis}

After $18 \mathrm{~d}$ of operation, the output voltage became stable and implied that the anodic biofilm were matured and ready for bacterial community analysis. Biofilm samples were collected from the anodes surface after the anodes were rinsed with ultra-pure sterile. Bacterial DNA samples were obtaine using a powersoil DNA isolation kit (MO Bio Laboratories, USA) and the collected DNA samples were further purified with a kit (BioTeke, China). The universal primers of 27F (5'-AGAGTTTGATCCTGGCTCAG-3') and 533R (5' -TTACCGCGGCTGCTGGCAC-3') were adopted for the amplifica- tion of the V3 region of the bacterial 16S rRNA gene. The PCR amplification procedure followed a protocol in our previous report [24]. The resulted amplicons were then quantitated with QuantiFluor ${ }^{\text {TM}}$-ST for further high-throughput sequencing.

Low-quality sequences were removed with QIIME software. The qualified sequences were clustered into operational taxonomic units (OTUs) at 0.03 sequence distance using MOTHUR. Based on the clusters, the number of OTUs, Chao1 index of the rarefaction curves, and phylogenetic richness index for diversity were calculated to determine the diversity and richness of each sample.

\section{Results and Discussion}

\subsection{Effect of Burial Depth of Anode On Voltage Output}

Time-course variations of the voltage in SMFC-5, SMFC-10 and SMFC-15 with an external resistance of $1000 \Omega$ were shown in Fig. 2. It was shown that the voltage output increased quickly in the initial $7 \mathrm{~d}$, and exhibited a peak voltage of 385, 432, 465 $\mathrm{mV}$ for SMFC-5, 10, and 15, respectively. Subsequently the voltage decreased and reached a steady state, with an average voltage of 318, 345 and $394 \mathrm{mV}$ for SMFC-5, SMFC-10 and SMFC-15, respectively. The stable voltage output indicated that exoelectronges were successfully enriched on the anode surface [25, 26]. The effect of anode depth on the voltage output was in line with a prior research [1]. The performance of SMFC-15 was better than SMFC-5 and SMFC-10, which implied that the physical and chemical characteristics of deeper sediment favored the metabolism of anodophiles or electrophiles [14, 15].

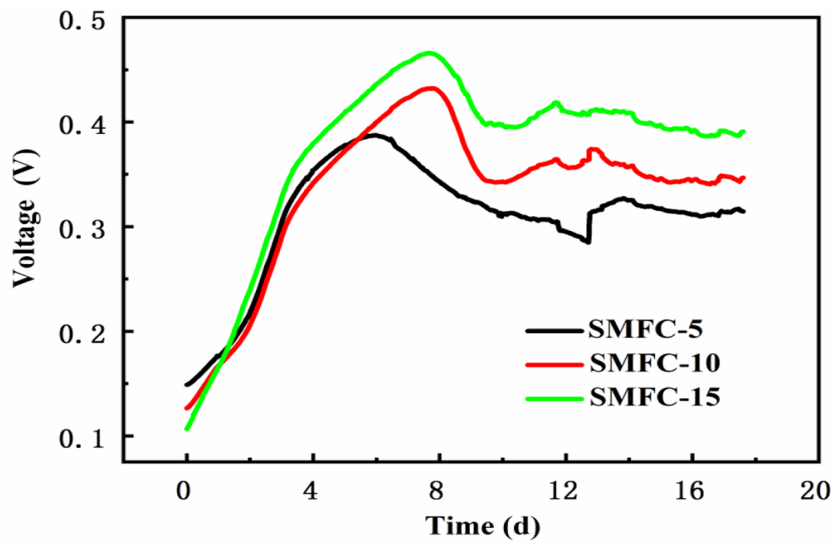

Fig. 2. Voltage output of the three SMFCs with differently embedded depth anode during a 18-d operation.

Table 1. Pyrosequencing Information of SMFC-5, SMFC-10, SMFC-15 and Sediment

\begin{tabular}{lccccc}
\hline \multirow{2}{*}{ Sample } & \multirow{2}{*}{$\begin{array}{c}\text { Effective } \\
\text { sequences }\end{array}$} & OTUs & Chao 1 & $\begin{array}{c}\text { Phylogenetic } \\
\text { diversity }\end{array}$ & Shannon \\
\hline SMFC-5 & 3820 & 1438 & 6271 & 124.45 & 8.65 \\
SMFC-10 & 3710 & 1275 & 5008 & 117.89 & 8.35 \\
SMFC-15 & 3093 & 1005 & 3457 & 97.33 & 8.27 \\
Sediment & 4165 & 2134 & 10022 & 172.52 & 10.18 \\
\hline
\end{tabular}




\subsection{Richness and Diversity of Phylotypes}

Overall pyrosequencing information and alpha diversity of the bacterial communities are summarized in Table 1. The number of the OTUs obtained was 1438, 1275, 1005 and 2134 for SMFC-5, SMFC-10, SMFC-15 and the original sediment, respectively. The result indicated that SMFC operation and increased burial depth of anode could decrease the microbial diversity. In addition, the maximum number of OTUs were determined as 6271 (SMFC-5), 5008 (SMFC-10) and 3457 (SMFC-15), suggesting that the samples located at deeper depth presented lower richness. The rarefaction curves showed increasing tendency even as the sequencing reads was larger than 3500, which implied that novel microbial phylotypes existed in the bacteria samples (Fig. 3).

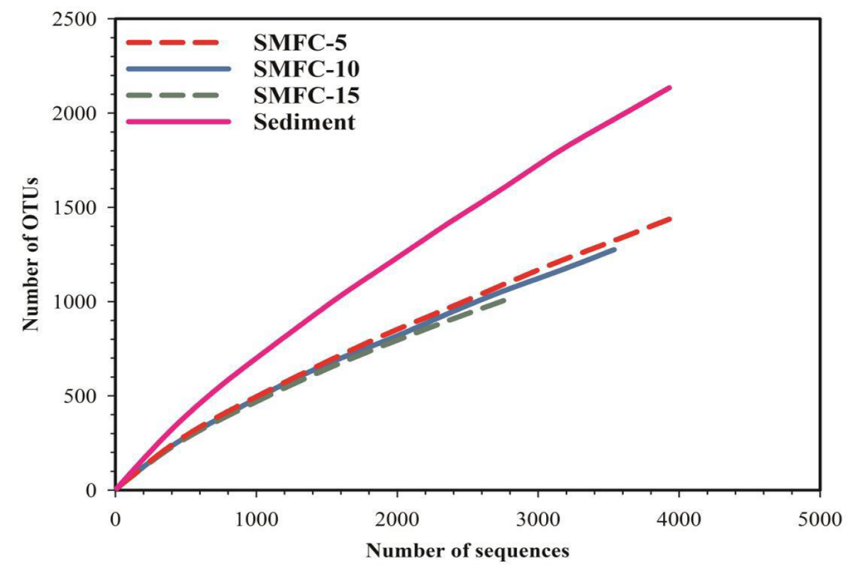

Fig. 3. Rarefaction curves based on pyrosequencing of SMFC-5, SMFC-10, SMFC-15 and raw sediment bacterial communities. The OTUs were defined by $3 \%$ distance.

\subsection{Bacterial Community Structures}

Hierarchical cluster analysis was conducted to reveal the relationship between the bacterial community structures (Fig. 4(a)). It was demonstrated that SMFC-5 and SMFC-10 were clustered together and well separated from SMFC-15 and the sediment. The results were further confirmed by the weighted principal coordinated anal- ysis, for which the components of 1, 2 and 3 accounted for $27.90 \%$, $20.90 \%$, and $18.17 \%$ of total community variation, respectively (Fig. 4b). The results suggested that burial depth of anode was an important factor to distinct the bacterial community structure of anodic biofilm in SMFCs.

\subsection{Phylotype Distribution}

To compare the phylotype distribution of the bacteria samples, the sequences were identified at the levels of phylum, class and order. As shown in Fig. 5, eight dominant bacterial phyla were revealed with a relative abundance $>2 \%$. Proteobacteria has dominated all the biofilms, followed by Bacteroidetes and Firmicutes. They accounted for 72.91\%, 78.86\%, 60.69\% and 58.64\% in SMFC-5, SMFC-10, SMFC-15 and sediment, respectively. Bacteroidetes accounted for $13.19 \%$ in SMFC-15, higher than $2.42 \%$ in SMFC-5, $2.88 \%$ in SMFC-10, and $5.62 \%$ in original sediment respectively. It was revealed that some members of Bacteroidetes demonstrate the ability of electron transfer to extracellular electron acceptors such as solid anode. The results implied that deeper depth might be beneficial for the accumulation of Bacteroidetes, which enhanced the performance of SMFCs. Chloroflexi is a common filamentous bacteria in wastewater treatment processes, which were abundant in the original sediment $(6.10 \%)$.

The identification of the bacterial communities at class level in SMFC-5, SMFC-10, SMFC-15 and sediment was illustrated in Fig. 6. Pyrosequencing detected that the majority of sequences belonged to 10 classes. Betaproteobacteria, out of Proteobacteria was determined as the most abundant class. Gammaproteobacteria, another member of Proteobacteria, was revealed as the sub-abundant class in the anodic biofilm, while Clostridia as typical cellulose degradation in a mesophilic environment was the secondly abundant in original sediment (10.78\%). Many bacteria out of Gammaproteobacteria, like Shewanella, Citrobacter, Aeromonas, Klebsiella and Pseudomonas, were widely known dominance in MFCs because of their ability to transfer electrons to anodes.

\subsection{Characterization of Dominant OTUs}

16S rRNA gene libraries were constructed based on the pyrosequencing results. As shown in Table 1, 5861 OTUs were classified with
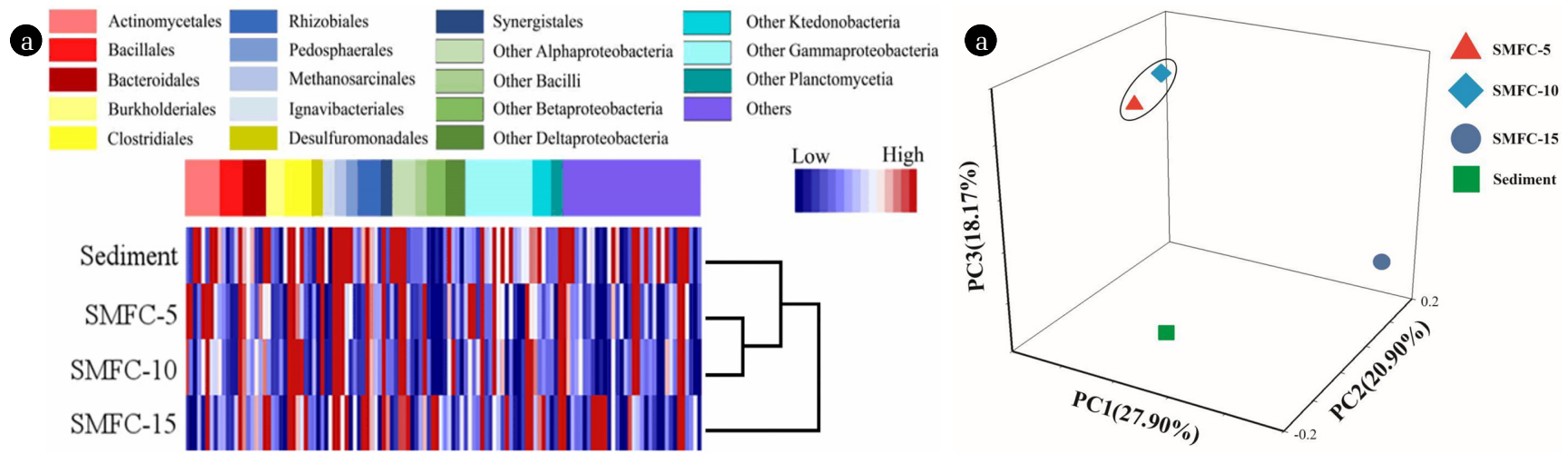

Fig. 4. (a) Hierarchical cluster analysis of bacterial communities of SMFC-5, SMFC-10, SMFC-15 and sediment. The OTUs were ordered by family. The bacterial communities were clustered based on $3 \%$ distance. The relative abundance of OTUs is reflected by the color of the scale. (b) Weighted principal coordinated analysis of bacterial communities of SMFC-5, SMFC-10, SMFC-15 and sediment. 


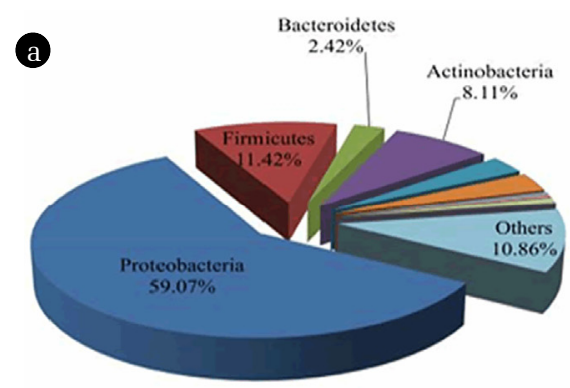

SMFC-5

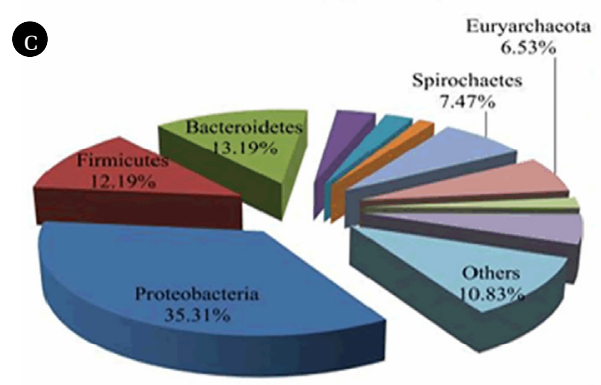

SMFC-15

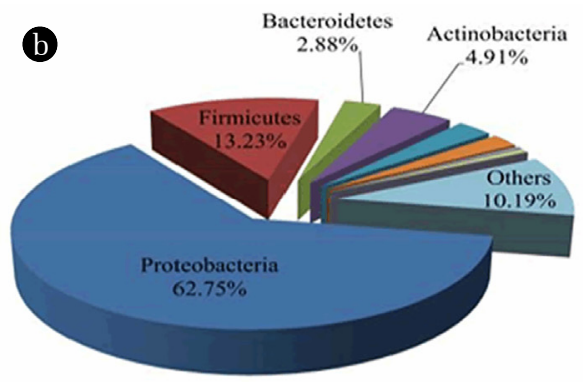

SMFC-10

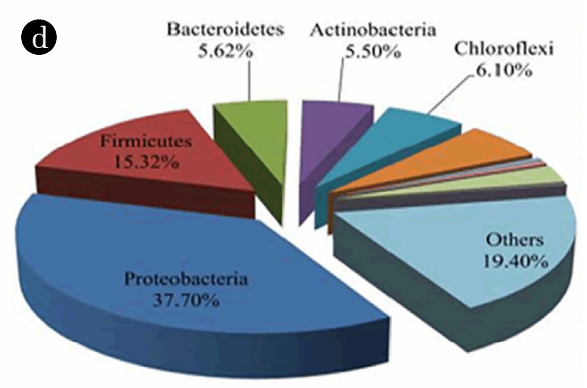

Sediment

E Protcobacteria = Firmicutes $\equiv$ Bacteroidetes $=$ Actinobacteria $=$ Chloroflexi $=$ Acidobacteria

$=$ Spirochaetes $\equiv$ Euryarchacota $=$ Planctomycetes $\equiv$ Synergistetes $\equiv$ Others

Fig. 5. Relative abundance of bacterial community composition at phylum levels in (a) SMFC-5, (b) SMFC-10, (c) SMFC-15 and (d) sediment.
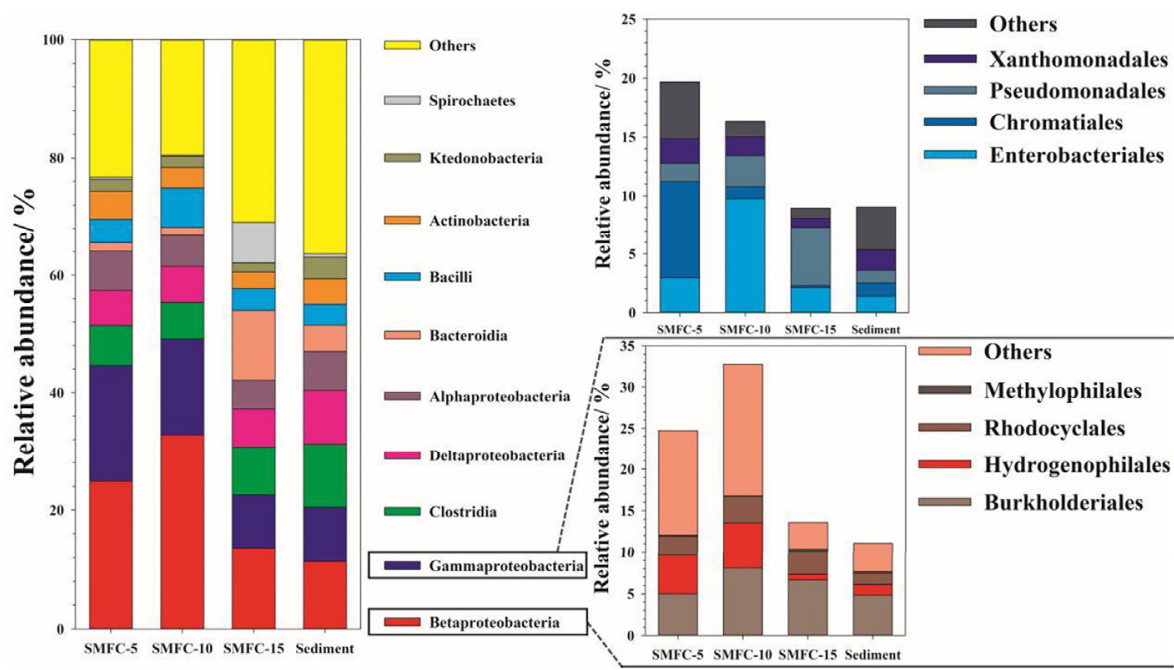

Fig. 6. Relative abundance of bacterial community composition at class and order levels in SMFC-5, SMFC-10, SMFC-15 and sediment.

a $3 \%$ distance. Out of the 5861 OTUs, only 115 OTUs were shared by the 4 samples. Moreover, 23 OTUs with a relative abundance larger than $0.5 \%$ were determined as dominant OTUs and accounted for $13.37-33.94 \%$ of the total sequences (Fig. 7(a)). Out of the 23 dominant OTUs, there were 6,3 and 6 OTUs belonged to the classes of Betaproteobacteria, Deltaproteobacteria, and Gammaproteobacteria, respectively, while only 1 dominant OTU belonged to Bacteroidia, Bacilli, Clostridia, Spirochaetes, and Synergistia. Among the 23 dominant OTUs, three OTUs (2328, 884 and 6493) had a relative abundance larger than 5\% in SMFC-10. The results demonstrated that only few species were dominant in the biofilm samples. The relative identifications of the dominant OTUs according to the results of BLAST were illustrated in Fig. 7(b).There were 19 GenBank matches belonging to cultured bacteria, and the other 4 OTUs were unclassified.

The OTUs had different relative abundance in the four microbial communites. The OTU-11403, as the most abundant one in SMFCS-15 (6.50\%), rarely appeared in SMFCS-5 (0.026\%) and sedi- 

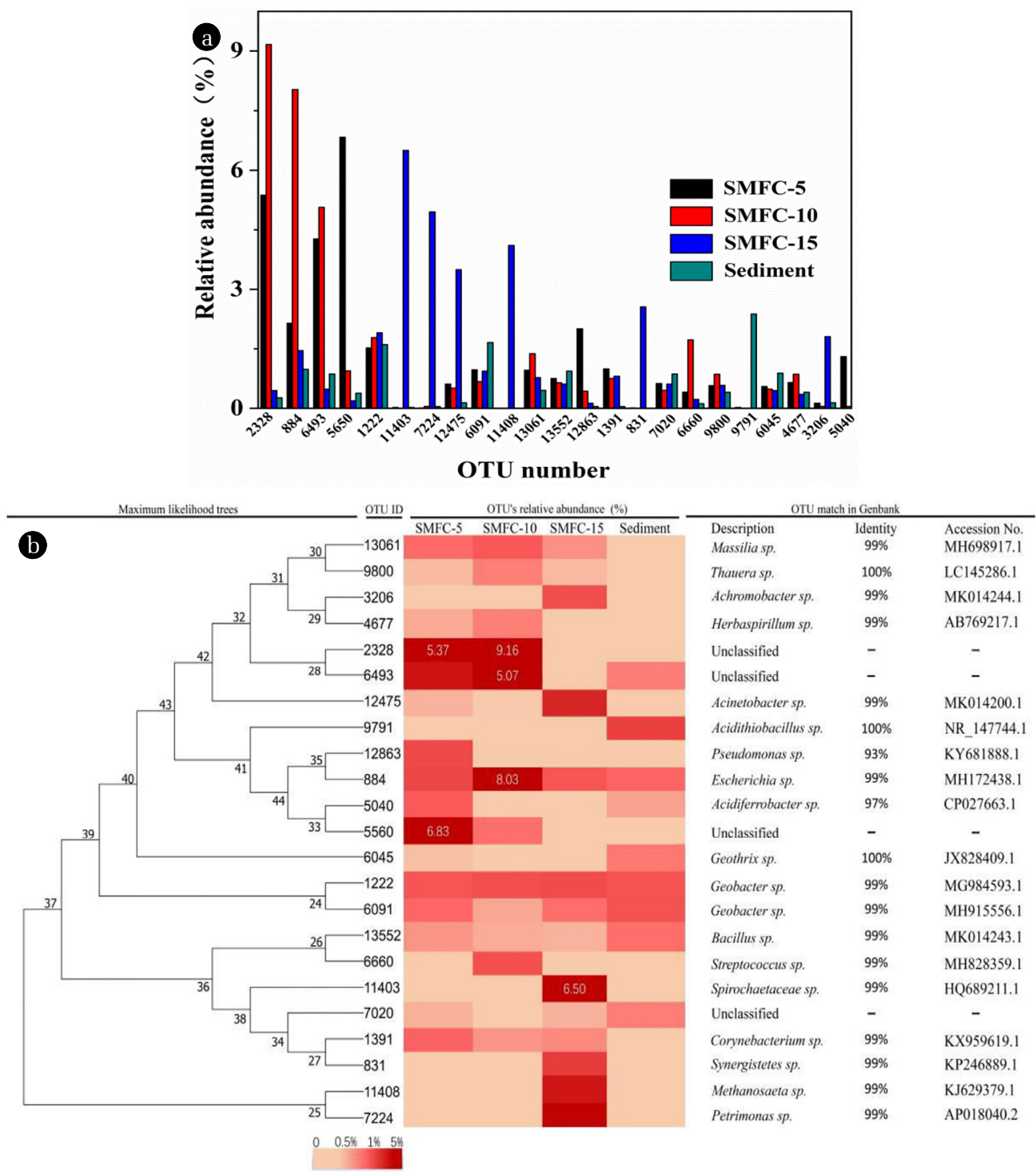

Fig. 7. (a) Relative abundance of the dominant OTUs in SMFC-5, SMFC-10, SMFC-15 and sediment. (b) Identification of the dominant OTUs. The color intensity in each panel shows the relative abundance.

ment (0.024\%), while not found in SMFCS-10. The results indicated that OTU-11403 had higher adaptability in the deeper sediment. The OTU-11403 was phylogenetically related (99\% identity) to Spirochaetaceae sp. A previous study demonstrated that Spirochaetaceae had appeared in anodic biofilm of an MFC using dairy manure as substrate [27]. The results indicated that burial depth was an influencing factor to shape the microbial community structure.

Electrochemically active bacteria were an indispensable portion of the SMFC, OTU-1222 and 609 showed similarities of $99 \%$ to Geobacter sp. Geobacter sulfur reduces strain is one of the most widely reported electrochemically active bacteria and is known as the model strain for studying extracellular electron transfer mechanism [28, 29]. Besides OTU-1222 and 609, OTUs 12863 was 99\% identical to Pseudomonas sp which was also reported as an electroactive bacterium [30, 31].

\section{Conclusions}

The results demonstrated that the anode installed deeper, produced a higher voltage. The indexes of phylotype richness and phylogenetic diversity of bacteria in biofilm attached to anode were negatively correlated to the burial depth of the anode. Betaproteobacteria and Gammaproteobacteria are more abundant in deeper depth (10 and $15 \mathrm{~cm}$ ). The electroactive microbes of Spirochaetaceae sp. had the largest abundance in the biofilm attached to anode embedded at $15 \mathrm{~cm}$ depth. These results further clarified the relationship between current generation and microbial community structure related to location depth of anode in SMFCs, and thus providing valuable information to improve the performance of SMFCs from the perspective of microbial community structure. 


\section{Acknowledgment}

This work was supported by Natural science foundation of Fujian Province (2019J01848), Xiamen Science and Technology Plan Guidance Project (3502Z20179029), and Scientific Climbing Plan of Xiamen University of Technology (XPDKQ18032).

\section{Author Contributions}

YC.W. (Ph.D.) conducted all the experiments and wrote the manuscript. HJ.W. (Bachelor) analyzed the partial data. HY.F. (Professor) and ZN.D. (Ph.D.) revised the manuscript. ZJ.W. (Associate Professor) wrote and revised the manuscript.

\section{References}

1. An J, Kim B, Nam J, Ng HY, Chang IS. Comparison in performance of sediment microbial fuel cells according to depth of embedded anode. Bioresour. Technol. 2013;127:138-142.

2. Reimers CE, Tender LM, Fertig S, Wang W. Harvesting energy from the marine sediment-water interface. Environ. Sci. Technol. 2001;35:192-195.

3. Shantaram A, Beyenal H, Veluchamy RRA, Lewandowski Z. Wireless sensors powered by microbial fuel cells. Environ. Sci. Technol. 2005;39:5037-5042.

4. Donovan C, Dewan A, Heo D, Lewandowski Z, Beyenal H. Sediment microbial fuel cell powering a submersible ultrasonic receiver: new approach to remote monitoring. J. Power Sources. 2013;233:79-85.

5. Zhang F, Tian L, He Z. Powering a wireless temperature sensor using sediment microbial fuel cells with vertical arrangement of electrodes. J. Power Sources. 2011;196:9568-9573.

6. Gong Y, Radachowsky SE, Wolf M, Nielsen ME, Girguis PR, Reimers CE. Benthic microbial fuel cell as direct power source for an acoustic modem and seawater oxygen/temperature sensor system. Environ. Sci. Technol. 2011;45:5047-5053.

7. Wu SS, Deng H, Cheng H, Liu L, Zhong WH. A novel sediment microbial fuel cell based sensor for on-line and in situ monitoring copper shock in water. Electroanalysis 2018;30:2668-2675.

8. Zhao Y, Li Z, Ma J. Enhanced bioelectroremediation of a complexly contaminated river sediment through stimulating electroactive degraders with methanol supply. J. Hazard. Mater. 2018;349:168-176.

9. Yan Z, Song N, Cai H, Tay JH, Jiang H. Enhanced degradation of phenanthrene and pyrene in freshwater sediments by combined employment of sediment microbial fuel cell and amorphous ferric hydroxide. J. Hazard. Mater. 2012;199:217-225.

10. Li X, Wang X, Zhang Y, et al. Salinity and conductivity amendment of soil enhanced the bioelectrochemical degradation of petroleum hydrocarbons. Sci. Rep. 2016;6:1-11.

11. Reimers CE, Tender LM, Fertig S, Wang W. Harvesting energy from the marine sediment-water interface. Environ. Sci. Technol. 2016;35:192-195.

12. Wang HM, Luo HP, Fallgren $\mathrm{PH}$, Jin S, Ren ZJ. Bioelectrochemical system platform for sustainable environ- mental remediation and energy generation. Biotechnol. Adv. 2015;33:317-334.

13. Zabihallahpoor A, Rahimnejad M, Talebnia F. Sediment microbial fuel cells as a new source of renewable and sustainable energy: present status and future prospects. Rsc. Adv. 2015;5: 94171-94183.

14. DiChristina TJ. Effects of nitrogen and nitrite in dissimilatory ion reduction by Shewanella putrefaciens 200. J. Bacteriol. 1992;174:1891-1896.

15. Froelich PN, Klinkhammer GP, Bender ML, et al. Early oxidation of organic matter in pelagic sediments of the eastern equatorial Atlantic: Suboxic diagenesis. Geochim. Cosmochim. Ac. 1979;43: 1075-1090.

16. Boyue L, Min J, Hongyan Z. Anodic potentials, electricity generation and bacterial community as affected by plant roots in sediment microbial fuel cell: Effects of anode locations. Chemosphere 2018;209:739-747.

17. Armato C, Ahmed D, Agostino V, Traversi D, Traversi R, Tommasi T. Anodic microbial community analysis of microbial fuel cells based on enriched inoculum from freshwater sediment Bioproc. Biosyst. Eng. 2019;26: 26829-26843.

18. Zhao Q, Li R, Ji M. Organic content influences sediment microbial fuel cell performance and community structure. Bioresour. Technol. 2016;220:549-556.

19. Xu X, Zhao Q, Wu M. Biodegradation of organic matter and anodic microbial communities analysis in sediment microbial fuel cells with/without Fe(III) oxide addition. Bioresour. Technol. 2016;225:402-408.

20. Deng H, Wu YC, Zhang F. Factors affecting the performance of single-chamber soil microbial fuel cells for power generation. Pedosphere 2014;24:330-338.

21. Quince C, Lanzén A, Curtis TP, et al. Accurate determination of microbial diversity from 454 pyrosequencing data. Nat. Methods. 2009;6:639-641.

22. Lu L, Xing DF, Ren NQ. Pyrosequencing reveals highly diverse microbial communities in microbial electrolysis cells involved in enhanced $\mathrm{H} 2$ production from waste activated sludge. Water Res. 2012;46:2425-2434.

23. Xiao Y, Zheng Y, Wu S, Yang ZH, Zhao F. Bacterial community structure of autotrophic denitrification biocathode by 454 pyrosequencing of the 16S rRNA gene. Microb. Ecol. 2015;69:492-49.

24. Wang ZJ, Zheng Y, Xiao Y, et al. Analysis of oxygen reduction and microbial community of air-diffusion biocathode in microbial fuel cells. Bioresour. Technol. 2013;144:74-79.

25. Song TS, Jiang HL. Effects of sediment pretreatment on the performance of sediment microbial fuel cells. Bioresour. Technol. 2011;102:10465-10470.

26. Song TS, Yan ZS, Zhao ZW, Jiang HL. Removal of organic matter in freshwater sediment by microbial fuel cells at various external resistances. J. Chem. Technol. Biotechnol. 2010;85: 1489-1493.

27. Zhang CP, Chen SS, Liu GL. Characterization of strain. Pseudomonas sp. Q1 in microbial fuel cell for treatment of quinoline contaminated water. Pedosphere. 2012;22:528-535.

28. Bond DR, Lovley DR. Electricity production by Geobacter sulfurreducens attached to electrodes. Appl. Environ. Microb. 2003;69: 1548-1555. 
29. Lovley DR. Electromicrobiology. Annu. Rev. Microbiol. 2012;66: 391-409.

30. Rabaey K, Boon N, Siciliano SD. Biofuel cells select for microbial consortia that self-mediate electron transfer. Appl. Environ.
Microb. 2004;70:5373-5382.

31. Zhang F, Zhao Q, Jiao Y. Biocathode microbial fuel cell for efficient electricity recovery from dairy manure. Biosens. Bioelectron. 2012;31:537-543. 